\title{
Seropositividad a Herpesvirus Canino tipo 1 en canes con antecedentes de problemas reproductivos en criaderos de Lima
}

\section{Seropositivity to Canine Herpesvirus 1 in dogs with history of reproductive problems in kennels in Lima}

\author{
Valeria Machuca $^{1,2}$, Luis M. Jara ${ }^{1}$, Luisa Echevarría ${ }^{1}$
}

\section{Resumen}

\begin{abstract}
El propósito del estudio fue evaluar la frecuencia serológica del Herpesvirus canino tipo 1 (HVC-1) en canes con antecedentes de problemas reproductivos (infertilidad, reabsorción embrionaria, abortos y mortinatos) en criaderos de Lima, Perú. Se obtuvieron 40 muestras de suero en 2019 de canes de nueve criaderos. Se determinó la presencia de anticuerpos anti-HVC-1 mediante un kit comercial de ELISA indirecto. Veintiséis muestras fueron positivas (65\%), estando el 37.5\% (9/24) de las hembras positivas en estro en el momento de la toma de muestra. Se reporta una exposición alta frente a HVC-1 en criaderos de Lima junto con una seropositividad de ocho veces el punto de corte de acuerdo al kit serológico utilizado.
\end{abstract}

Palabras clave: Herpesvirus Canino tipo 1, seropositividad, problemas reproductivos, criaderos

\section{AbStract}

The aim of this study was to evaluate the serological frequency of canine herpesvirus type $1(\mathrm{CHV}-1)$ in dogs with a history of reproductive problems (infertility, embryo reabsorption, abortions and stillbirths) in kennels in Lima, Peru. Forty serum samples

\footnotetext{
${ }^{1}$ Facultad de Medicina Veterinaria y Zootecnia, Universidad Peruana Cayetano Heredia, Lima, Perú

${ }^{2}$ E-mail:valeria.ast94@gmail.com
}

Recibido: 9 de noviembre de 2020

Aceptado para publicación: 12 de agosto de 2021

Publicado: 27 de octubre de 2021

CLos autores. Este artículo es publicado por la Rev Inv Vet Perú de la Facultad de Medicina Veterinaria, Universidad Nacional Mayor de San Marcos. Este es un artículo de acceso abierto, distribuido bajo los términos de la licencia Creative Commons Atribución 4.0 Internacional (CC BY 4.0) [https:// creativecommons.org/licenses/by/4.0/deed.es] que permite el uso, distribución y reproducción en cualquier medio, siempre que la obra original sea debidamente citada de su fuente original 
were obtained in 2019 from dogs from nine commercial kennels. The presence of antiCHV -1 antibodies was determined using a commercial indirect ELISA kit. Twenty-six samples were positive (65\%), with $37.5 \%(9 / 24)$ of positive females being in estrus at the time of sampling. A high exposure to CHV-1 is reported in kennels in Lima together with a seropositivity of eight times the cut-off point.

Key words: Canine Herpesvirus type 1, seropositivity, reproductive problems, kennels

\section{INTRODUCCIÓN}

El Herpesvirus Canino tipo 1 (HVC-1) se reportó por primera vez en 1965 en Nueva York, EEUU, en canes neonatos que presentaron muerte fulminante (Sykes, 2014). Este virus pertenece a la familia Herpesviridae, subfamilia Alphaherpesvirinae y afecta tanto canes domésticos como silvestres (Evermann et al., 1984). Es sensible a altas temperaturas, siendo su replicación óptima entre $35-37{ }^{\circ} \mathrm{C}$ y presenta un estado de latencia en las neuronas del ganglio trigémino y nódulos linfáticos retrofaríngeos (Galosi, 2007; Nauwynck, 2010). Su reactivación está asociada a periodos de estrés o inmunosupresión producidos por sobrepoblación de animales, hacinamiento, gestación y administración de corticosteroides (Evermann et al., 2011; Sykes, 2014).

El contagio se da por contacto venéreo y oronasal, así como por vía transplacentaria en neonatos (Ronsse et al., 2004). Las patologías asociadas se encuentran relacionadas a la reproducción, mayormente infertilidad, reabsorciones embrionarias, abortos, mortinatos y lesiones papovesiculares genitales (Ronsse et al., 2004; Evermann et al., 2011; Rezaei et al., 2020). Sin embargo, también se han registrado casos de traqueobronquitis, blefaritis, conjuntivitis y queratitis ulcerativa y no ulcerativa (Ledbetter et al., 2009). La enfermedad en neonatos dura entre 1 y 3 días (Carmichael, 2004) y se caracteriza por signos clínicos como anorexia, disnea, dolor abdominal, hipotermia, incoordinación, heces blandas amarillo ver- dosas y descarga nasal serosa o hemorrágica, acompañado de hemorragias multifocales en órganos, así como petequias en mucosas y, posteriormente, la muerte (Poulet et al., 2001; Ronsse et al., 2004).

El HVC-1 se encuentra distribuido a nivel mundial, con prevalencias serológicas que pueden llegar hasta el $88 \%$ según el área geográfica (Decaro et al., 2008). La mayor frecuencia de casos se observa en criaderos de canes, donde se sugiere la coexistencia de factores condicionantes tanto ambientales como inmunológicos (Van Gucht et al., 2001). La prevalencia en países de América del Sur no ha sido claramente definida (Góngora et al., 2007). El único estudio realizado en Lima Metropolitana en canes con antecedentes de problemas reproductivos reportó 32\% de seropositividad (Góngora et al., 2007). Sin embargo, el aumento de crianza de canes de raza en criaderos propios o comerciales, donde se obtiene un elevado número de camadas requiere determinar el nivel de exposición al HVC-1. En ese contexto, el presente estudio tuvo como objetivo determinar la presencia de anticuerpos anti HVC-1 en perros con antecedentes de problemas reproductivos de criaderos de Lima utilizando una prueba comercial de ELISA indirecto.

\section{Materiales y Métodos}

Se incluyó a nueve criaderos formales de canes de Lima, ubicados en los distritos de Los Olivos, Chorrillos, Lurín y Chilca. El 
muestreo fue por conveniencia y se realizó en los meses de abril a junio de 2019. Se muestrearon 40 canes con edades entre 6 meses hasta 10 años (tres cachorros: 6-9 meses; tres jóvenes: 9-18 meses; 34 adultos y gerontes: 18-120 meses). Cinco canes fueron machos y 35 hembras (12 en etapa de estro). Las razas fueron American Bully (5), American Staffordshire Terrier (18), Boxer (2), Bulldog Inglés (3), Bulldog Francés (3), Bull Terrier (3), Pastor Alemán (4), Rottweiler (1) y Schnauzer miniatura (1). Los animales no tenían antecedentes de vacunación contra HVC-1, sin embargo, presentaron al menos un antecedente reproductivo como infertilidad (incapacidad de preñez), reabsorciones embrionarias (interrupción del desarrollo embrionario detectado por ultrasonografía), abortos, crías mortinatas o de animales cuyas madres tuvieron un episodio abortivo o eran sobrevivientes de camadas con mortalidad.

Se tomó una muestra de sangre $(3 \mathrm{ml})$ en tubos sin anticoagulante que fue centrifugada a $2500 \mathrm{rpm}$ por 10 minutos para recuperar el suero. El procesamiento de las muestras se realizó en el Laboratorio de Reproducción Animal de la Facultad de Medicina Veterinaria y Zootecnia, Universidad Peruana Cayetano Heredia, Lima, Perú.

Las muestras de suero fueron analizadas mediante la técnica de ELISA indirecto para detectar anticuerpos frente HVC-1, según el protocolo del kit comercial «Canine Herpes Virus ELISA» (XpressBio ${ }^{\circledR}$, USA). Las densidades ópticas (DO) de la reacción colorimétrica se registraron en un espectrofotómetro (ELx800, Biotek, USA), mediante el software Gen 5 v. 2.04 (Biotek, USA). Una muestra se consideró positiva si la diferencia entre la absorbancia de la muestra en el pocillo tapizado y en el pocillo sin tapizar con el antígeno viral resultó $>0.3$.

Los resultados se colocaron en tablas de frecuencia y se utilizó estadística descriptiva. Se evaluó además la correlación de la edad (meses) de los animales seropositivos con la densidad óptica (DO) obtenida mediante la prueba de Pearson. El nivel de significancia fue de 0.05 y se analizó con el programa IBM SPSS Statistics 25.0.

Cuadro 1. Frecuencia de hembras seropositivas a Herpesvirus canino Tipo 1 (HVC1) según problema reproductivo $(n=24)$

\begin{tabular}{lccc}
\hline \multirow{2}{*}{ Problema reproductivo } & Canes & \multicolumn{2}{c}{ Canes seropositivos a } \\
& muestreados & \multicolumn{2}{c}{ HVC-1 } \\
\cline { 2 - 4 } & $(\mathrm{n})$ & $\mathrm{n}$ & $\%$ \\
\hline Aborto & 13 & 13 & 54.1 \\
Infertilidad & 5 & 3 & 12.5 \\
Mortinatos & 4 & 3 & 12.5 \\
Reabsorción embrionaria & 2 & 1 & 4.1 \\
Reabsorción embrionaria e infertilidad & 3 & 3 & 12.5 \\
Infertilidad, aborto y mortinatos & 1 & 1 & 4.1 \\
Sobreviviente de camada con alta & 4 & 0 & 0 \\
mortalidad & & & 0 \\
Sobreviviente de madre con episodio & 3 & 0 & 0 \\
abortivo & & & \\
\hline
\end{tabular}




\section{Resultados}

La frecuencia de seropositividad frente a HVC-1 en canes con antecedentes de problemas reproductivos provenientes de criaderos de Lima fue de $65.0 \%$ (26/40). La media de los valores de DO en las muestras positivas fue de $2.42 \pm 0.63$. De los animales que resultaron seropositivos, 24 correspondieron a hembras y 2 a machos.

La correlación entre la intensidad de la seropositividad (densidad óptica) y la edad de los animales resultó positiva, pero con un coeficiente bajo (0.13), no significativo. El problema reproductivo más frecuente registrado entre las hembras seropositivas fue el aborto $(54.1 \%, 13 / 24)$, seguido de infertilidad (Cuadro 1). De otra parte, el 37.5\% (9/24) se encontraba en estro al momento de la toma de muestras.

\section{Discusión}

La primera y única evidencia publicada de HVC-1 en Lima fue reportada por Góngora et al. (2007) mediante inmunofluorescencia indirecta en canes mestizos, quienes encontraron una frecuencia serológica del $32 \%$; es decir, la mitad de la hallada en el presente estudio. No obstante, los animales provenientes de criaderos son de raza y podrían estar más expuestos a diversos agentes infecciosos, debido a una mayor interacción en situaciones de riesgo con restos placentarios, descargas vulvares o cachorros posiblemente infectados, entre otros factores (Ronsee et al., 2004). Sin embargo, la raza no representaría un factor que influye en una mayor seropositividad (Ronsee et al., 2004; Evermann, 2005). De otra parte, Van Gucht et al. (2001) demostraron una relación significativa entre la seroprevalencia con casos de mortalidad neonatal y complicaciones para la preñez.
Si bien todos los animales del estudio tenían antecedentes de problemas reproductivos, 35\% (14/40) fueron seronegativos a HVC-1, pudiendo haber estado afectados por otros agentes infecciosos como Brucella canis, Mycoplasma/Ureaplasma o Campylobacter (Givens y Marley, 2008). Asimismo, es necesario determinar causas no infecciosas como hipoluteoidismo, estrés y deficiencias nutricionales, entre otras (Verstegen et al., 2008).

Se debe considerar que, en un estado de latencia, el virus puede reactivarse sobre todo cuando las hembras están en estro o durante la gestación (Anvik, 1991), condiciones relacionadas a estados de estrés o inmunosupresión (Okuda et al., 1993; Greene y Ford, 2012; Sykes, 2014). Si bien los estudios serológicos pueden determinar exposición a virus, no identifican un estado de enfermedad activa o de diseminación del agente (Yapici et al., 2018); sin embargo, cabe resaltar que los valores de DO de los animales seropositivos fueron altos ( 8 veces el punto de corte), lo cual haría suponer títulos altos.

Por otro lado, dos de los canes machos que resultaron seropositivos en el presente estudio tuvieron como antecedente de problema reproductivo la infertilidad, además de haber tenido historial de cruce con hembras. Este hallazgo sería de consideración ya que existe un potencial riesgo de transmisión sexual del virus debido a que los machos suelen ser utilizados para cubrir varias hembras entre diferentes criadores.

En el presente estudio se encontró una correlación positiva baja entre la intensidad de la seropositividad y edad de los animales, resultado no concluyente debido al número pequeño de muestras y a que el número de canes por grupo etario no fue homogéneo. Los perros jóvenes y los cachorros resultaron seronegativos, posiblemente debido a que aún no han iniciado una etapa reproductiva y, por tanto, de mayor exposición (Ronsse et al., 2004). 
Ronsee et al. (2004) reportaron fluctuaciones en los títulos de anticuerpos antiHVC-1 con respecto a la etapa del ciclo estral, encontrando títulos más elevados en hembras en estro. Sin embargo, considerando los resultados obtenidos en el presente estudio, se sugiere que el análisis de exposición de las hembras debería ser evaluado durante todo el ciclo estral. Esto fue una limitante en el presente estudio, además de haber incluido un reducido número de animales. No obstante, se reporta por primera vez evidencia de seroreactividad a HVC-1 en canes provenientes de criaderos de Lima. Los hallazgos demuestran una exposición alta al virus en animales con antecedentes de problemas reproductivos, por lo que se sugiere tomar en consideración medidas de vigilancia, manejo y prevención en criaderos.

\section{Conclusiones}

La frecuencia serológica frente a Herpesvirus canino Tipo 1 (HVC-1) en canes con antecedentes de problemas reproductivos en criaderos de Lima Metropolitana fue alta $(65.0 \%)$.

\section{Literatura Citada}

1. Anvik J. 1991. Clinical considerations of canine herpesvirus infection. Vet Med 86: 394-403.

2. Carmichael L. 2004. Neonatal viral infections of pups: canine herpesvirus and minute virus of canines (Canine Parvovirus-1) In: Carmichael L (ed). Recent advances in canine infectious diseases. Ithaca, NY: International Veterinary Information Service. [Internet]. Available in: https://www.ivis.org/ library/recent-advances-canineinfectious-diseases/neonatal-viralinfections-of-pups-canine

3. Decaro N, Martella V, Buonavoglia C. 2008. Canine adenoviruses and herpesvirus. Vet Clin North Am Small Anim
Pract 38: 799-814. doi: 10.1016/ j.cvsm.2008.02.006

4. Evermann JF, LeaMaster B, McElwain T, Potter K, McKeiman A, Green J. 1984. Natural infection of captive coyote pups with a Herpesvirus antigenically related to canine Herpesvirus. J Am Vet Med Assoc 185: 1288-1290.

5. Evermann JF. 2005. Canine herpesvirus infection: update on risk factors and control measures. Vet Forum 69: 32-37.

6. Evermann JF, Ledbetter E, Maes R. 2011. Canine reproductive, respiratory, and ocular diseases due to canine herpesvirus. Vet Clin N Am-Small 41: 1097-1120. doi: 10.1016/j.cvsm.2011.08.007

7. Galosi CM. 2007. Herpesvirus canino 1: agente etiológico y enfermedad. Analecta Vet 27: 28-35.

8. Góngora V, Sandoval N, Manchego A. 2007. Evidencia serológica de la presencia del virus Herpes Canino Tipo 1 en la provincia de Lima. Rev Inv Vet Perú 18: 154-157. doi: 10.15381/rivep.v18i2.1488

9. Givens D, Marley M. 2008. Infectious causes of embryonic and fetal mortality. Theriogenology 70: 270-285. doi: 10.1016/j.theriogenology.2008.04.018

10. Greene C, Ford R. 2012. Canine herpesvirus infection. In: Infectious diseases of the dog and cat. USA: Elsevier. p 48-65.

11. Ledbetter E, Dubovi E, Kim S, Maggs D, Bicalho. 2009. Experimental primary ocular canine herpesvirus-1 infection in adult dogs. Am J Vet Res 70: 513-21. doi: 10.2460/ajvr.70.4.513

12. Nauwynck H. 2010. Canine herpes virus 1 - infections in dogs: truth and lies. In: VII Congress of the European Veterinary Society for Small Animal Reproduction (EVSSAR).

13. Okuda Y, Ishida K, Hashimoto A, Yamaguchi T, Fukushi H, Hirai K, et al. 1993. Virus reactivation in bitches with a medical history of herpesvirus infection. Am J Vet Res 54: 551-554. 
14. Poulet H, Guigal P, Soulier M, Leroy V, Fayet G, Minke J, Chappuis G. 2001. Protection of puppies against canine herpesvirus by vaccination of the dams. Vet Rec 148: 691-695. doi: 10.1136/vr.148.22.691

15. Rezaei M, Jajarmi M, Alizadeh R, Khalili M, Babaei H. 2020. First molecular study of Canine Herpesvirus1 in reproductive specimens of adult dogs in southeast of Iran. Com Immunol Microbiol Infect Dis 17: 101487. doi: 10.1016/j.cimid.2020.101487

16. Ronsse V, Verstegen J, Onclin K, Farnir F, Poulet H. 2004. Risk factors and reproductive disorders associated with canine herpesvirus-1 (CHV-1). Theriogenology 61: 619-636. doi: 10.1016/s0093-691x(03)00249-8
17. Sykes J. 2014. Canine herpesvirus infection. In: Davidson A (ed). Canine and feline infectious diseases. USA: Elsevier. p 166-169.

18. Van Gucht S, Nauwynck H, Pensaert $M . ~ 2001$. Prevalence of canine herpesvirus in kennels and the possible association with fertility problems and neonatal death. Vlaams Diergen Tijds 70: 204-211.

19. Verstegen J, Dhaliwal G, VerstegenOnclin K. 2008. Canine and feline pregnancy loss due to viral and noninfectious causes: a review. Theriogenology 70: 304-19: doi: 304-319.10.1016/ j.theriogenology.2008.05.035

20. Yapici O, Avci O, Levent O, Hasircioglu S. 2018. Detection of canine herpesvirus infection on dogs. Microbiol Res J Int 24: 1-6. doi: 10.9734/MRJI/ 2018/419 